$\mathrm{P}-\mathrm{\square}$

\title{
Matrix assisted laser desorption/ionization mass spectrometry imaging method for visualizing spatial distribution of synthetic polymers combining with Kendrick mass defect analysis
}

\author{
Takaya Satoh, ${ }^{1}{ }^{1} *$ Yoshihisa Ueda, ${ }^{1}$ \\ ${ }^{1}$ JEOL Ltd,3-1-2 Musashino, Akishima, Tokyo, Japan \\ *corresponding author's e-mail: taksatoh@jeol.co.jp
}

(Received: May 30, 2019; Accepted: July 23, 2019)

\begin{abstract}
Matrix assisted laser desorption/ionization (MALDI) mass spectrometry is a powerful tool for the analysis of synthetic polymers. This technique can determine molecular weight distributions, which are often expressed as number average molecular weight $\left(\mathrm{M}_{n}\right)$, weight average molecular weight $\left(\mathrm{M}_{\mathrm{w}}\right)$ and dispersity (D). We have investigated a imaging mass spectrometry (MSI) visualization method for synthetic polymers that uses $M_{n}, M_{w}$, and D as indices. We also applied the Kendrick mass defect (KMD) analysis to improve this method. By using this method, several dozen to hundred of mass images corresponding to each polymer's degree of polymerization were integrated into four mass images related to the total ion intensity, $M_{n}, M_{w}$, and D. As a result, the polymer distributions on sample surface become much easier to overview.
\end{abstract}

\section{Introduction}

Matrix assisted laser desorption/ionization (MALDI) mass spectrometry is a powerful tool for the analysis of synthetic polymers. This technique can determine molecular weight distributions, which are often expressed as number average molecular weight $\left(\mathrm{M}_{\mathrm{n}}\right)$, weight average molecular weight $\left(\mathrm{M}_{\mathrm{w}}\right)$ and dispersity (D). MALDI mass spectrometry imaging (MSI) is a method to investigate the spatial distribution of target compounds by scanning the laser irradiation point of mass spectra acquisition. It is most commonly applied to biological applications, such as proteins, peptides, lipids, metabolites and administered drugs. The distributions of target compounds were shown by extracting the mass images for specific molecular weights. However, given the molecular weight distributions for synthetic polymers, the mass images based on specific mass peaks, which means specific degrees of polymerization, will not express the spatial distribution of entire polymer series. In this report, we have investigated a MSI visualization method for synthetic polymers that uses $M_{n}, M_{w}$, and $D$ as indices.
We also applied the Kendrick mass defect (KMD) analysis to improve this method[1]. KMD analysis is an effective method to overview the polymer series in mass spectra. It can easily find polymer series in mass spectra even for minor components. We have used KMD analysis for grouping polymer series before visualizing polymer distributions. We also used it for comparing the mass spectra between characteristic regions identified by visualization of polymers. As a result, it became easier to express the polymer distribution on a sample surface using MALDI-MSI.

\section{Experiment}

We prepared following polymer solutions diluted in methanol $(10 \mathrm{mg} / \mathrm{mL})$ (See Table1). Afterwards, $\alpha$-CHCA matrix and NaTFA cationization agent solutions were mixed with the polymer solutions before spotting them onto the target plate. We made two types of samples. For sample 1(See figure 1a), the left spot included only PEG 1000, and the right spot included a mixture of PEG 600 and 1000. For Sample2 (See figure 1b), the first spot included PEG and PEG-C12H25 in 
the ratio of 10:1 (v/v), and the second spot included PEG and PPG in the ratio of 10:1 (v/v). The diameters of the spots were about $2 \mathrm{~mm}$. MALDI-MSI was performed over the area that included both spots. Mass spectra and MSI data were acquired using a high-mass resolution MALDI time-of-flight mass spectrometer (JMS-S3000, JEOL Ltd.). The laser was a Nd:YLF solid-state laser $(349 \mathrm{~nm})$ and laser spot diameter adjusted to $20 \mu \mathrm{m}$.

\begin{tabular}{|l|l|}
\hline Polymers & Molecular weight \\
\hline Polyethylene glycol (PEG) & $600,1000,2000$ \\
\hline $\begin{array}{l}\text { Polyethylene glycol monododecyl } \\
\left.\text { ethere (PEG- } \mathrm{C}_{12} \mathrm{H}_{25}\right)\end{array}$ & 1100 \\
\hline Polypropylene glycol (PPG) & 2000 \\
\hline
\end{tabular}

Table 1 Sample polymers.

Sample1 :

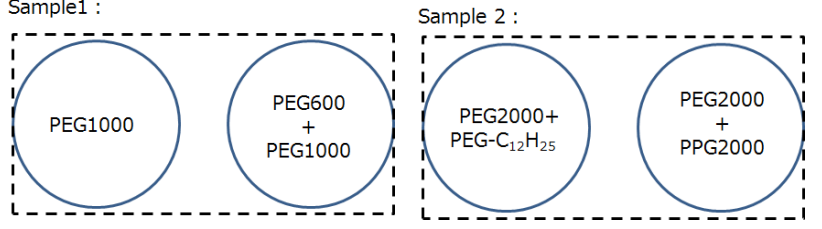

Figure 1 Two type of samples. Dotted lines and solid lines are corresponded to boundaries of measurement areas and sample spotted areas.

\section{Results}

To visualize the spatial distribution of the polymers, the total intensity (TIC), $\mathrm{M}_{\mathrm{n}}, \mathrm{M}_{\mathrm{w}}$, and D were calculated for each pixel (see figure 2 for workflow). These values were all calculated by using the following procedure. i) Data acquisition, ii) create average mass spectrum, iii) specified polymer series, iv) extract mass images from raw data according to the polymer series peak lists, v) The values of TIC, $M_{n}, M_{w}$ and D for each polymer series are calculated for each pixel and vi) Draw images of TIC, $M_{n}, M_{w}$, and D. The calculation equation used in step v) is

$$
\begin{aligned}
& \text { TIC }=\Sigma \text { Int }_{\mathrm{i}} \\
& \mathrm{M}_{\mathrm{n}}=\Sigma\left(\mathrm{M}_{\mathrm{i}} \times \mathrm{Int}_{\mathrm{i}}\right) / \mathrm{Int}_{\mathrm{i}} \\
& \mathrm{M}_{\mathrm{w}}=\Sigma\left(\mathrm{M}_{\mathrm{i}}^{2} \times \mathrm{Int}_{\mathrm{i}}\right) /\left(\mathrm{M}_{\mathrm{i}} \times \mathrm{Int}_{\mathrm{i}}\right) \\
& \mathrm{D}=\mathrm{M}_{\mathrm{w}} / \mathrm{M}_{\mathrm{n}}
\end{aligned}
$$

where, $\mathrm{M}_{\mathrm{i}}$ and $\mathrm{Int}_{\mathrm{i}}$ indicated the molecular weight and ion intensity of the degree of polymerization $i$, respectively.

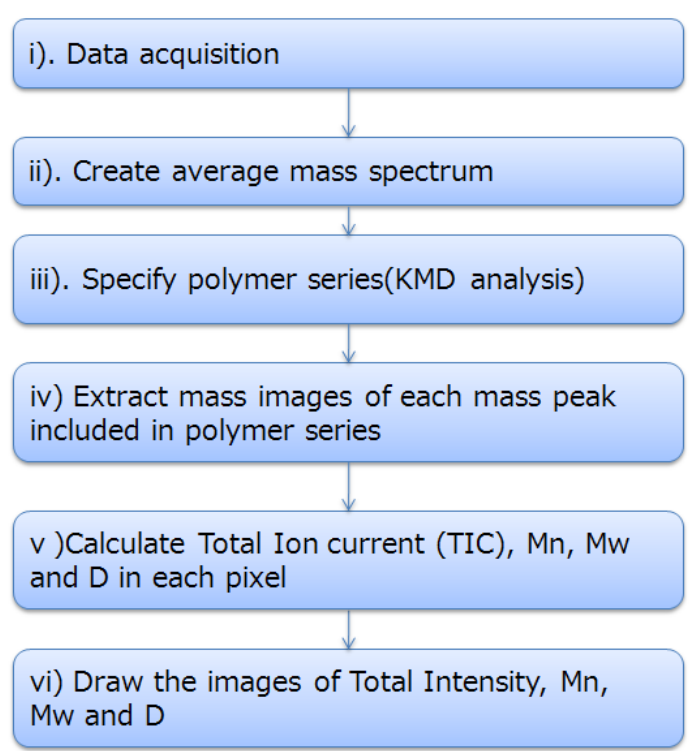

Figure 2 Work flows for making images related to TIC, $M_{n}$, $\mathrm{M}_{\mathrm{w}}$, and D.

The images related to TIC, $\mathrm{M}_{\mathrm{n}}, \mathrm{M}_{\mathrm{w}}$, and D for Sample1 are shown in Figure 3. Several dozen to hundred of mass images corresponding to each polymer's degree of polymerization were integrated into four mass images related to the TIC, $M_{n}, M_{w}$, and D. By doing this, the polymer spatial distributions for the different polymer samples were much easier to view in the images.

We are planning to present the MALDI-MSI measurement on degraded polymers using ultraviolet lays, where the polymer distribution and end-group have been changed, were also analyzed using this method. a) TIC

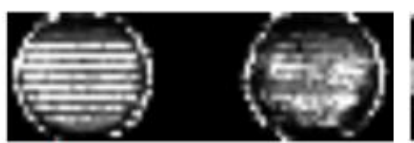

c) $\mathrm{Mw}$

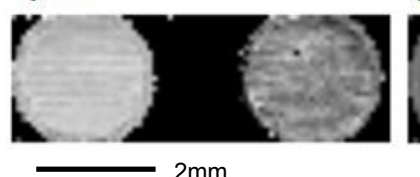

$2 \mathrm{~mm}$ b) $\mathrm{Mn}$

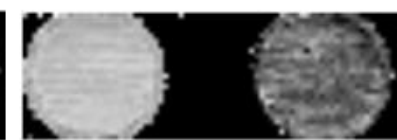

d) D

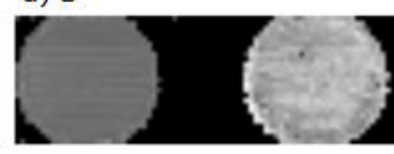

Figure 3 The images related to a) TIC, b) $M_{n}$, c) $M_{w}$, and d) D for Sample1.

\section{Reference}

[1] H. Sato, S. Nakamura, K. Teramoto, T. Sato, J. Am. Soc. Mass Spectrom. 25, 1346 (2014) 